$\xi$

\title{
Slip Velocity and Temperature Jump on Dissipative CASSON Fluid with CATTANEO-CHRISTOV Heat Flux Model: Spectral Relaxation Method
}

\author{
K. Gangadhar ${ }^{1}$, K. V. Ramana ${ }^{2}$, T. Kannan ${ }^{3}$ and B. Rushi Kumar ${ }^{4 *}$ \\ ${ }^{1}$ Department of Mathematics, Acharya Nagarjuna University, Ongole, Andhra Pradesh-523001,India \\ ${ }^{2}$ Deparment of Mathematics, NRI Institute of Technology, Guntur, Andhra Pradesh-522438, India \\ ${ }^{3}$ Deparment of Mathematics, K.S.R College of Technology,Tiruchengode-637215, Tamil Nadu, India \\ ${ }^{4}$ Department of Mathematics, School of Advanced Sciences, VIT, Vellore-632014,TN, India \\ *Corresponding authorE-mail: rushikumar@vit.ac.in
}

\begin{abstract}
A numerical analysis is performed for investigating the slip flow of a viscous dissipative Casson fluid towards a stretching sheet with Cattaneo-Christov heat flux and variable viscosity. The nonlinear partial differential equations are transformed with appropriate similarity variables into a system of nonlinear ordinary differential equations. Numerical solutions are carried out by using efficient Spectral relaxation method. Notable accuracy of the present results has been obtained with previous results in a limiting sense from the literature. It is found that thermal relaxation time has an inverse relationship with the fluid temperature. Interestingly, the fluid velocity is gradually decreasing with higher values of slip factor.
\end{abstract}

Keywords: Cattaneo-Christov heat flux model, Stretching sheet, Temperature Jump, MHD, Slip velocity.

\section{Introduction}

The study of heat transfer over a stretching sheet has applications in various industrial and engineering processes. Many processing industries such as paper production, food processing hot rolling, glass-fabric, and purification of crude oil plays an important role for governing momentum and heat transfer boundary layer flow for a stretching sheet [1-3]. The heat conduction law proposed by Fourier [4] has been the source to predict the heat transfer behavior in different realistic situations. One of the most important flaws of this model is it produces a parabolic energy equation means an initial disturbance would instantly affect the system under assumption. Several modified versions of the Fourier's law have been introduced by many researchers ([5-7]). The Fourier's law with the inclusion of relaxation time for heat flux is revised by Cattaneo [8]. For forced temperature gradient, time is required to establish the steady heat conduction. Christov [9] obtained a material invariant formulation of the Cattaneo's model during the consideration of Oldroyd's upper-convected derivative. Straughan [10] employed Cattaneo-Christov model to report the thermal convection in an incompressible flow. Ciarletta and Straughan [11] discussed the structural stability and uniqueness of CattaneoChristov equations. Han et al. [12] applied Cattaneo-Christov model to compute the slip flow and the heat transfer of viscoelastic fluid bounded by a stretching plate. The boundary layer theory of Cattaneo-Christov heat flux model to viscoelastic fluid flow over an exponentially stretched surfacing is investigated by Khan et al. [13]. Malik et al. [14] examined the effect of variable viscosity and MHD on a boundary layer flow in Casson fluid with Cattaneo-Christov heat flux model. They found that temperature distribution within the boundary layer increases more rapidly in Fou- rier's law case than Cattaneo-Christov heat flux model. The boundary layer flow between two stretched rotating disks with Cattaneo-Christov heat flux model is studied by Hayat et al. [15]. The thermally stratified stretching boundary layer flow with Cattaneo-Christov heat flux model is numerically investigated by Hayat et al. [16].

Most of the industrial fluids exhibits a non-Newtonian fluid behavior such as paper making, processing of food, drilling of petroleum products, slurry transporting, ceramic processing, blood and . Casson fluid is a type of non-Newtonian fluid model and it is one of the most commonly used rheological models such as blood, chocolate, juices etc. Casson [17] considered the validity of the Casson fluid model in his studies about the flow characteristics of blood. The boundary layer flow of a Casson fluid to a heat transfer towards an exponentially stretching surface in presence of thermal radiation is studied by Nadeem et al. [18]. Mukhopadhyay et al. [19] reported the two-dimensional flow over unsteady stretching surface. The boundary layer in a Casson fluid and a heat transfer through a nonlinear stretching surface is carried out by Mukhopadhyay [20]. Nadeem et al. [21] illustrated the three-dimensional steady flow of Casson fluid past a porous linear stretching sheet. Mukhopadhyay et al. [22] numerically studied the steady boundary layer flow and a heat transfer in the Casson fluid over exponentially stretching permeable surface with prescribed heat flux. Mahanta et al. [23] examined the effects of MHD three-dimensional Casson fluid pass a porous linear stretching sheet. The boundary layer flow for a Casson nanofluid convinced by nonlinear stretching surface is discussed by Mustafa et al. [24]. Animasaun et al. [25] explored the boundary layer flow to steady incompressible laminar free convective magneto-hydrodynamic (MHD) Casson fluid. Das et al [26] analyzed heat transfer to unsteady Casson fluid in a vertical plate. The heat and mass transfer of Casson fluid 
over an exponentially permeable stretching sheet is reported by Raju et al. [27]. An idea of generalized Couette flow for an incompressible Casson fluid between the same plates utilizing the same boundary condition is illustrated by Ramesh et al. [28]. A free convection flow of the Casson fluid over a fluctuating vertical plate with constant wall temperature is carried out by Khalid et al. [29]. The MHD stagnated point flow of Casson nano fluid over a non linear stretching sheet with the velocity slip and the convective boundary conditions is numerically examined by Ibrahim et al. [30]. Sethetal [31] investigated about erects of Joule dissipation and Navier's partial slip on hydromagnatic thin film flow of a Casson fluid embedded in a non-Darcy porous medium. Shateyi et al. [32] reported the free convective flow of a Casson fluid over an unsteady permeable stretching surface with viscous dissipation effect.

The effect of viscous dissipation has a significant impact on vigorous natural or mixed convection processes. Gebhart [33] initiated a number to represent an effect of viscous dissipation in the system. An effect of the viscous dissipation parameter on the finite amplitude B'enard convection is examined by Turcotte et al. [34]. Barletta et al. [35] studied the stability of a flow was induced in viscous dissipation. The impact of viscous dissipation in a porous medium with the horizontal temperature gradient along the upper boundary and an adiabatic lower boundary is reported by Barletta et al. [36]. The effect of viscous dissipation on convection on the flow in a Darcy medium is illustrated by Barletta and Nield [37]. The impact of viscous dissipation on the double diffusive convection occurring solely due to the viscous dissipation is carried out by Barletta and Nield [38] and reexamined by Roy and Murthy [39] with Soret effect. Khader and Mziou [40] studied the boundary layer flow of a viscoelastic fluid over a permeable stretching surface with viscous dissipation effect using Chebyshev spectral method. An impact of viscous dissipation on the convective instability induced by inclined temperature gradients in a non-parcy porous medium with a horizontal throw flow was presented by Roy and Murthy [41]. The effects of viscous dissipation and uniform heat source/sink on MHD boundary layer flow over a stretching sheet numerically investigated by Metri et al. [42]. Palani et al. [43] examined the effects of viscous dissipation, MHD and heat source on free convective flow with variable surface temperature past a semi-infinite vertical plate.

Inspired by the above-mentioned studies, it is noted that steady temperature jump and velocity slip flow of a viscous dissipative Casson fluid towards a stretching sheet with variable thickness has not been discussed so far. The viscosity of the fluid is presumed to be erratic with temperature. Cattaneo-Christov heat flux model is employed in this present work to investigate the insight of heat transfer phenomena. The transformed differential equations are solved numerically by using Spectral relaxation method. The effects of the governing parameters of the problem are discussed and studied.

\section{Mathematical Formulation}

Consider a steady, incompressible two-dimensional boundary layer flow of viscous dissipative Casson fluid over stretching sheet with variable thickness. Assume that the velocity of sheet be, $U_{w}=U_{0}(x+b)^{m}$, where $U_{0}$ denotes reference velocity. It is considered that the wall thickness of the stretching sheet may increase or decrease with distance from slot by varying the power index $\mathrm{m}$. For $m=1$ the problem reduces to flat stretching sheet. The viscosity of the fluid is assumed to be variable i.e. $\mu=\mu_{0} e^{T-T_{\infty}}$. Cattaneo-Christov heat flux model is employed to explore the heat transfer characteristics instead of Fourier's law. Cartesian coordinates are chosen in such a way that $x$-axis is along the along the variable sheet and $y$-axis is perpendicular to the sheet.

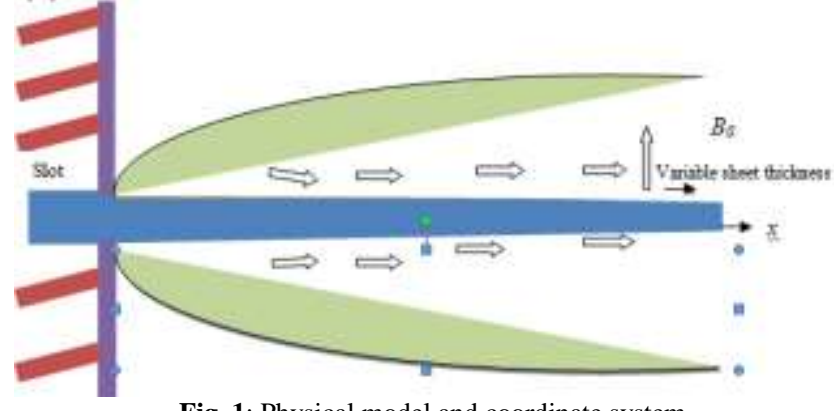

Fig. 1: Physical model and coordinate system

The magnetic field of strength $B_{0}$ is applied normal to the sheet

$$
\nabla \cdot V=0
$$

$\rho \frac{D V}{D t}=\nabla \cdot \tau+\rho b$

where $\tau$ is Cauchy stress tensor, $\rho$ is the density, $\frac{D}{D t}$ is material time derivative, $\mathrm{V}$ is the velocity field, and $\nabla$ is an operator and $b=J \times B \rho$ is the body force.

The stress tensor is defined as

$$
\tau=\left\{\begin{array}{l}
\left\{2\left(\mu_{B}+\frac{p_{z}}{\sqrt{2 \pi}}\right) e_{i j}, \pi>\pi_{c}\right. \\
\left\{2\left(\mu_{B}+\frac{p_{z}}{\sqrt{2 \pi}}\right) e_{j i}, \pi<\pi_{c}\right.
\end{array}\right.
$$

where $\pi$ is the product of the component of rate of deformation rate itself i.e. $\pi=e_{i j} e_{j i} e_{i j}$ indicates the $(i, j)$ component of the deformation rate, $\pi_{c}$ is the critical value of $\pi$ based on nonNewton model, $p_{z}$ is the yield stress on the field and $\mu_{B}$ is a plastic dynamic viscosity of the non-Newtonian fluid. By employing the boundary layer approximation, the conservation law of mass, momentum and energy equations takes the form:

$$
\frac{\partial u}{\partial x}+\frac{\partial v}{\partial y}=0
$$

$u \frac{\partial u}{\partial x}+v \frac{\partial u}{\partial y}=\frac{\mu}{\rho_{0}}\left(1+\frac{1}{\beta}\right) \frac{\partial^{2} u}{\partial y^{2}}+v\left(1+\frac{1}{\beta}\right) \frac{\partial u}{\partial y} \frac{\partial \mu}{\partial y}-\frac{\sigma B_{0}^{2}}{\rho} u$,

$\rho c_{p} V . \nabla T=-\nabla \cdot q+\left(1+\frac{1}{\beta}\right) \varphi$

where $T$ is the temperature of the fluid, $u$ and $v$ are the velocity components along $\mathrm{x}$ and $\mathrm{y}$ directions respectively, $B_{0}$ is the magnitude of magnetic field, $v$ is the kinematic viscosity, $\rho$ is the density, $c_{p}$ is the specific heat, $\beta$ is the Casson parameter, $\varphi$ is the dissipative function, $q$ is the heat flux and $\mu_{\text {is }}$ the variable viscosity.

From equation (6)

$$
q+\lambda_{2}\left(\frac{\partial q}{\partial t}+V \cdot \nabla \cdot q-q \cdot \nabla V+(\nabla \cdot V) q\right)=-k \nabla T+\left(1+\frac{1}{\beta}\right) \tau: \nabla V
$$


where $k$ the thermal conductivity of the fluid, $\lambda_{2}$ is the thermal relaxation time and $\tau$ is the extra stress.

From equations (6) and (7):

$$
\begin{aligned}
& u \frac{\partial T}{\partial x}+v \frac{\partial T}{\partial y}+\lambda_{2}\left(\begin{array}{l}
u \frac{\partial u}{\partial x} \frac{\partial T}{\partial x}+v \frac{\partial u}{\partial y} \frac{\partial T}{\partial y} \\
+u \frac{\partial v}{\partial x} \frac{\partial T}{\partial y}+v \frac{\partial u}{\partial y} \frac{\partial T}{\partial x} \\
+2 u v \frac{\partial^{2} T}{\partial x \partial y}+u^{2} \frac{\partial^{2} T}{\partial x^{2}}+v^{2} \frac{\partial^{2} T}{\partial y^{2}}
\end{array}\right) \\
& =\frac{k}{\rho c_{p}} \frac{\partial^{2} T}{\partial y^{2}}+\frac{v}{c_{p}}\left(1+\frac{1}{\beta}\right)\left(\frac{\partial u}{\partial y}\right)^{2}
\end{aligned}
$$

The boundary conditions :

$$
\begin{aligned}
& u=U_{w}(x)+L_{1} \frac{\partial u}{\partial y}=U_{0}(x+b)^{m}+L_{1} \frac{\partial u}{\partial y}, v=0 \\
& T=T_{w}+D_{1} \frac{\partial T}{\partial y} \text { at } y=A(x+b)^{\frac{1-m}{2}} \\
& u \rightarrow 0, T \rightarrow T_{\infty} \text { as } y \rightarrow \infty
\end{aligned}
$$

where $D_{1}$ the temperature jump coefficient and $L_{1}$ is the slip velocity coefficient.

The similarity transformations are

$$
\begin{aligned}
& u=U_{0}(x+b)^{m} F^{\prime}(\eta), \\
& v=-\sqrt{\frac{(m+1) v U_{0}(x+b)^{(m-1)}}{2}}\left[F(\eta)+\eta \frac{m-1}{m+1} F^{\prime}(\eta)\right], \\
& \eta=\sqrt{\frac{(m+1) U_{0}(x+b)^{m-1}}{2 v}} y, \theta(\eta)=\frac{T-T_{\infty}}{T_{w}-T_{\infty}}, \\
& \psi=\sqrt{\frac{2 v U_{0}(x+b)^{m+1}}{m+1}} F(\eta)
\end{aligned}
$$

By using the above transformations, the governing equations are changed as follows:

$$
\begin{aligned}
& \left(1+\frac{1}{\beta}\right)(1-B \theta)\left(F^{\prime \prime \prime}-B \theta^{\prime} F^{\prime \prime}\right)+F F^{\prime \prime}-\frac{2 m}{m+1}\left(F^{\prime}\right)^{2}-M p F^{\prime}=0 \\
& \theta^{\prime \prime}+\operatorname{Pr} F \theta^{\prime}+\operatorname{Pr} \gamma\left(\frac{m-3}{2} F F^{\prime} \theta^{\prime}-\frac{m+1}{2} F^{2} \theta^{\prime \prime}\right)+\operatorname{Pr} E c\left(1+\frac{1}{\beta}\right) F^{\prime \prime 2}=0
\end{aligned}
$$

$F\left(\alpha_{1}\right)=\frac{\alpha_{1}(1-m)}{(1+m)}, F^{\prime}\left(\alpha_{1}\right)=1+\alpha_{2} F^{\prime \prime}(0), \theta(0)=1+\alpha_{3} \theta^{\prime}(0)$,

$F^{\prime}(\infty) \rightarrow 0, \theta(\infty) \rightarrow 0$

where $\alpha_{1}=A\left(\sqrt{\frac{U_{0}(m+1)}{2 v}}\right)$ denotes the plate surface.

The transformed equations are

$$
\begin{aligned}
& \left(1+\frac{1}{\beta}\right)(1-\gamma \theta)\left(f^{\prime \prime \prime}-\gamma \theta^{\prime} f^{\prime \prime}\right)+f f^{\prime \prime}-\frac{2 m}{m+1}\left(f^{\prime}\right)^{2}-M p f^{\prime}=0 \\
& \theta^{\prime \prime}+\operatorname{Pr} f \theta^{\prime}+\operatorname{Pr} \lambda\left(\frac{m-3}{2} f f^{\prime} \theta^{\prime}-\frac{m+1}{2} f^{2} \theta^{\prime \prime}\right)+\operatorname{Pr} E c\left(1+\frac{1}{\beta}\right) f^{\prime \prime 2}=0
\end{aligned}
$$

The corresponding boundary conditions are $f(0)=\frac{\alpha_{1}(1-m)}{(1+m)}, f^{\prime}(0)=1+\alpha_{2} f^{\prime \prime}(0), \theta(0)=1+\alpha_{3} \theta^{\prime}(0)$

$f^{\prime}(\infty) \rightarrow 0, \theta(\infty) \rightarrow 0$.

where $\gamma=e^{-\zeta\left(T-T_{\infty}\right)} \quad$ the variable viscosity parameter, $M p=\frac{2 \sigma B_{o}{ }^{2}}{(m+1) \rho U_{0}^{2}(x+b)^{m-1}}$ is Hartmann number, $\operatorname{Pr}=\frac{\mu c_{p}}{k}$ is Prandtl number, $\lambda=\lambda_{2} U_{0}(x+b)^{m-1}$ is the thermal relaxation parameter, $\quad E c=\frac{U_{w}^{2}}{c_{p}\left(T_{w}-T_{\infty}\right)} \quad$ is the Eckert number, $\alpha_{2}=L_{1} \sqrt{\frac{(m+1) U_{0}(x+b)^{m-1}}{2 v}}$ is the slip velocity factor, $\alpha_{3}=D_{1} \sqrt{\frac{(m+1) U_{0}(x+b)^{m-1}}{2 v}}$ is the thermal jump factor.

The wall shear stress at the sheet is given below

$\tau_{w}=\mu\left(1+\frac{1}{\beta}\right) \frac{\partial u}{\partial y}$

The skin friction can be defined as

$C_{f}=\frac{\tau_{w}}{\sqrt{(m+1) U_{0}} 2 v}\left(\frac{1}{(x+b)^{\frac{3 m-1}{2}}}\right)$

While the dimensionless forms of skin friction

$C_{f} \sqrt{\operatorname{Re}_{x}}=\left[\left(1+\frac{1}{\beta}\right)(1-\gamma \theta) f^{\prime \prime}\right]_{\eta=0}$

$$
\text { where } \operatorname{Re}_{x}=\sqrt{\frac{U_{0}}{v}} x \text {. }
$$

\section{Solution of the Problem}

The transformed nonlinear equations (15)-(16) subject to the boundary conditions (17) are numerically solved by the Spectral Relaxation Method (SRM) (Motsa and Makukula [44] and Kameswaran et al. [45]). The SRM method uses the Gauss- Seidel approach to decouple the system of equations. In the system of SRM technique the iteration scheme is obtained as

$$
f_{r+1}^{\prime}=p_{r}, f_{r+1}(0)=\alpha_{1}\left(\frac{1-m}{1+m}\right)
$$

$$
\begin{array}{r}
\left(1+\frac{1}{\beta}\right)\left(1-\gamma \theta_{r+1}\right) p_{r+1}^{\prime \prime}+\left(f_{r+1}-\left(1+\frac{1}{\beta}\right)\left(1-\gamma \theta_{r+1}\right) \gamma \theta_{r+1}^{\prime}\right) p_{r+1}^{\prime} \\
-(M p) p_{r+1}=\frac{2 m}{m+1} p_{r+1}^{2}
\end{array}
$$

$$
\begin{aligned}
\left(1-\frac{m+1}{2} f_{r+1}^{2}\right) \theta_{r+1}^{\prime \prime}+\left(\operatorname{Pr} f_{r+1}+\operatorname{Pr} \lambda \frac{m-3}{2} f_{r+1} p_{r+1}\right) \theta_{r+1}^{\prime} & \\
& =-\operatorname{Pr} E c\left(1+\frac{1}{\beta}\right) p_{r+1}^{\prime 2}
\end{aligned}
$$

The boundary conditions for the above iteration scheme are 


$$
\begin{aligned}
& p_{r+1}(0)=1+\alpha_{2} p_{r+1}^{\prime}(0), \theta_{r+1}(0)=1+\alpha_{3} \theta_{r+1}^{\prime}(0) \\
& p_{r+1}(\infty)=0, \theta_{r+1}(\infty)=0
\end{aligned}
$$

The decoupled equations (21) - (25), are dealt by the Chebyshev spectral collocation method. The computational domain $[0, L]$ is changed to the interval $[-1,1]$ using $\eta=L(\xi+1) / 2$ on which the spectral method is applied. Here $L$ is used to invoke the boundary conditions at $\infty$. The crucial thought behind the spectral collocation technique is the introduction of a differentiation matrix $\mathcal{D}$ which is used to estimate the derivatives of the unknown variables at the collocation points as the matrix vector product of the form

$$
\frac{\partial f_{r+1}}{\partial \eta}=\sum_{k=0}^{N} D_{l k} f_{r}\left(\xi_{k}\right)=D f_{r}, l=0,1,2, \ldots \ldots \ldots . . N
$$

Where $N+1$ is the number of collocation points (grid points), $\mathrm{D}=2 \mathcal{D} / \mathrm{L}$, and $f=\left[f\left(\xi_{0}\right), f\left(\xi_{1}\right), f\left(\xi_{2}\right), \ldots \ldots \ldots f\left(\xi_{N}\right)\right]^{T}$ is represented the vector function at the collocation points. Higher-order derivatives are acquired as powers of $\mathrm{D}$, that is,

$$
f_{r}^{(p)}=D^{p} f_{r}
$$

where $p$ is the order of the derivative. By implementing the spectral method to equations (25)- (27), we get

$$
\begin{aligned}
& A_{1} f_{r+1}=B_{1}, f_{r+1}\left(\xi_{N}\right)=\alpha_{1}\left(\frac{1-m}{1+m}\right) \\
& A_{2} p_{r+1}=B_{2}, \alpha_{2} \sum_{k=0}^{\bar{N}} D_{\bar{N} k} p_{r+1}\left(\xi_{k}\right)-p_{r+1}\left(\xi_{k}\right)=-1, p_{r+1}\left(\xi_{0}\right)=0 \\
& A_{3} \theta_{r+1}=B_{3}, \alpha_{3} \sum_{k=0}^{\bar{N}} D_{\overline{N k}} \theta_{r+1}\left(\xi_{k}\right)-\theta_{r+1}\left(\xi_{k}\right)=-1, \theta_{r+1}\left(\xi_{0}\right)=0
\end{aligned}
$$

Here,

$$
\begin{aligned}
A_{1}= & D, B_{1}=p_{r+1} \\
A_{2}= & \left(1+\frac{1}{\beta}\right)\left(1-\gamma \theta_{r+1}\right) D^{2}+\operatorname{diag}\left(f_{r+1}-\left(1+\frac{1}{\beta}\right)\left(1-\gamma \theta_{r+1}\right) \gamma \theta_{r+1}^{\prime}\right) D-M p I \\
B_{2}= & \frac{2 m}{m+1} p_{r+1}^{2} \\
A_{3}= & \left(1-\frac{m+1}{2} f_{r+1}^{2}\right) D^{2} \\
& +\operatorname{diag}\left(\operatorname{Pr} f_{r+1}+\operatorname{Pr} \lambda \frac{m-3}{2} f_{r+1} p_{r+1}\right) D \\
B_{3}= & -\operatorname{Pr} E c\left(1+\frac{1}{\beta}\right) p_{r+1}^{\prime 2}
\end{aligned}
$$

From the equations (32)-(33), the identity matrix and diagonal matrix are denoted as $\mathrm{I}$ and $\mathrm{D}$, respectively, all of size $(N+1) \times(N+1)$ where $f, p$ and $\theta$ are the values of the functions respectively, when calculated at the grid points, $N$ represents the number of grid points and $\mathrm{r}$ denotes the iteration number. The Casson parameter is given by

$$
\beta=\frac{\mu_{B} \sqrt{2 \phi_{c}}}{P_{y}}
$$

Where $\mu_{B}$ plastic dynamic viscosity, $P_{y}$ is the yield stress of the fluid and $\phi_{c}$ is the critical value of $\phi$ which is product of the deformation rate with itself. For such fluid, only the initial shear threshold is reached, the fluid displaces linear share stress behaviour with respect to shear rate. It is clear from the expression that Casson factor is directly proportional to dynamic viscosity. Hence, enhance in $\beta$ means enhance in plastic dynamic viscosity $\mu_{B}$ which be a symbol of a higher resistance to the fluid flow and as outcome, a downfall is observed in the fluid velocity on increasing the Casson parameter.

The initial guesses to begin the SRM technique for equalities (24)(25) are taken as

$$
\begin{aligned}
& f_{0}(\eta)=\alpha_{1}\left(\frac{1-m}{1+m}\right)+\frac{1}{1+\alpha_{2}}\left(1-e^{-\eta}\right) \\
& p_{0}(\eta)=\frac{1}{1+\alpha_{2}} e^{-\eta}, \theta_{0}(\eta)=\frac{1}{1+\alpha_{3}} e^{-\eta}
\end{aligned}
$$

which are randomly selected functions that are satisfies the boundary conditions. The iteration is repeated until convergence attained. The convergence of the SRM strategy is indicated in terms of the infinity norm as

$$
E r=\operatorname{Max}\left(\left\|f_{r+1}-f_{r}\right\|,\left\|p_{r+1}-p_{r}\right\|,\left\|\theta_{r+1}-\theta_{r}\right\|\right)
$$

Accuracy of the method is established by increasing the number of collocation points $N$ until the solutions are consistent and moreover increases do not change the value of the solutions.

\section{Results and Discussion}

In order to get a clear insight of the physical problem, the velocity and temperature profiles have been discussed by assigning numerical values to the governing parameters encountered in the problem. Figure 2 shows that the flow behavior is affected along with the change in the Casson parameter $\beta$. When the Casson parameter is increased from 1 to $\infty$, the flow is progressively decreased. In Figure 3 it is noted that the temperature distribution increases inside the boundary layer with an increase in Casson fluid parameter $\beta$. Casson parameter has prompt the decrement in the velocity of fluid, thus, extra work done in dragging the fluid against the physical entries dissipates in the form of energy, and so increase in thermal boundary layer thickness is witnessed.

Figure 4 show how the velocity profile is affected by the magnetic field. On increasing $M$ causes fluid velocity inside the thin film as well as the film thickness is decreasing significantly. The interesting reason behind such an effect is that the magnetic field might be owed to induction of a retarding body force, is stated as Lorentz force, due to the presence of magnetic field in an electrically conducting thin film. The effects of a magnetic field significantly influences the temperature profile that is presented in figures 5. It is also observed that the temperature profile increases with increasing values of the magnetic field parameter.

The dependence of the axial velocity profile $f^{\prime}(\eta)$ on variable viscosity parameter $\gamma$ is illustrated in Figure 6. It is clearly witnessed that as $\gamma$ increases the boundary layer thickness decreases and the fluid velocity distribution is lowered notably. Physically, this is because a given larger fluid variable viscosity implies higher temperature difference between the surface and the ambient fluid. Hence, it can be concluded that an increase of $\gamma$, the temperature distribution throughout the boundary layer increases and it is 
shown in Figure 7. Figures 8 and 9 displays the impact of wall thickness parameter $\alpha_{1}$ on the velocity profile and temperature profile, respectively. It can be seen that for $m>1$, the velocity profile $f^{\prime}(\eta)$ decreases at any point near the stretched sheet as the wall thickness parameter increases, and for $m<1$ a significant decrease in velocity is observed. Moreover, it reveals that the increase of wall thickness parameter leads to thicker boundary layer. From Figure 9 shows that the wall thickness parameter reduced the temperature distribution and decreased the thickness of the boundary layer for $m<1$ where as reverse trend is noticed as

$m>1$. The flow velocity decreases for higher values of $\alpha_{1}$ and $m<1$ since under the variable wall thickness not all the pulling force of the stretching sheet can be transmitted to the fluid causing a reduce for both fiction between the fluid layers and temperature distribution for the fluid. However in the case of $m>1$ the velocity of the flow layers will increase causing enhance for the friction force between this layers and hence increasing its temperature. Figure 10 shows that, the presence of slip velocity within the boundary layer causes the velocity level along the sheet to decrease. For higher values of the velocity slip parameter the velocity distribution reduce near the surface of the sheet but it increments at larger distance. As slip occurs $\left(\alpha_{2} \neq 0\right)$, the flow of the stretching sheet becomes greater than the flow velocity near the sheet. Besides, increasing the values of velocity slip parameter $\alpha_{2}$ will considerably decrease the flow velocity because the condition of slip. From Figure 11 it is can be witnessed that not all the pulling force of the stretching sheet can be transmitted to the fluid, but the behavior of temperature distribution for the variation of the velocity slip parameter $\alpha_{2}$ is displayed. Also, it is seen that temperature and boundary layer thickness is higher for slip boundary when compared to the no-slip boundary $\left(\alpha_{2}=0\right)$. It is concluded that the slip parameter $\alpha_{2}$ should be assigned as minimum as much as possible in cooling systems.

The illustration of different thermal jump parameter $\alpha_{3}$ is presented in Figure 12. The increasing temperature jump parameter supports the decrease thermal boundary layer thickness. In this considered problem the temperature jump between the wall and the fluid indicates the thermal contact resistance which decreases the amount of heat exchange. It is also concluded that the slip velocity also has the similar influence on the velocity near the sheet. Moreover, with the increasing distance from the stretching sheet, the effect of temperature jump parameter on the temperature becomes smaller. Effects and influences of thermal time relaxation parameter $\lambda$ on temperature distribution are revealed in Figure 13. Temperature distribution within the boundary layer region decreases when thermal relaxation time parameter is increased. Actually when thermal time relaxation parameter is considerably high, then fluid particles need extra time in transferring the heat to its adjacent particles. For $\lambda=0$ the heat transfers with infinite speed through the whole material and hence temperature field is dominant for $\lambda=0$. The effects of viscous dissipation parameter $(E c)$ on the temperature profile are demonstrated in the Figure14. It represents the convection of kinematic energy into internal energy by work done against the viscous fluid stresses. Hence, higher viscous dissipative heat causes arise in the temperature, which is illustrated in Figure14. In Figure 15, the influence of Prandtl number $\mathrm{Pr}$ in the boundary layer on temperature profile is demonstrated. The numerical outcome reveals that an effect of increasing values of Prandtl number results in decreasing to the temperature and it is noted that decrease of thermal boundary layer thickness and in general lower average temperature within the boundary layer. Thus, in the case of smaller Prandtl number the boundary layer is thicker and the rate of heat transfer is reduced. Figure 16 shows that absolute values of the skin friction coefficient increases as in $M p$ increases. The negative sign of skin friction coefficient implies that the stretching sheet exerts a dragging force on the fluid and positive sign implies the opposite. In Figure 16, it is witnessed that by increasing $\beta$, the skin friction coefficient decreases. Figure 17 exhibits the influence of magnetic parameter and Casson parameter on Nusselt number. It reveals that increasing the magnetic parameter and Casson parameter lead to significant decrease in the local heat transfer rate.

In order to validate the accuracy of our numerical solution, we have compared our results with those of Wang [46], Salahuddin et al [47] and Malik et al [48]. Table 1 shows these values with those using the spectral relaxation method. This comparison is found to be in excellent agreement.

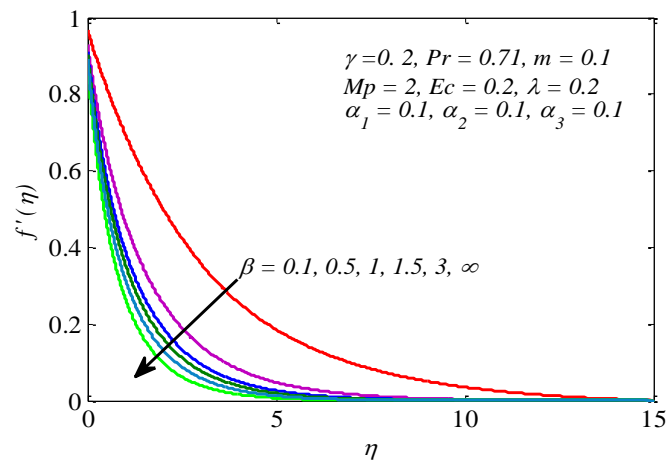

Fig. 2: Dimensionless velocity profiles $f^{\prime}(\eta)$ for the various values of $\beta$

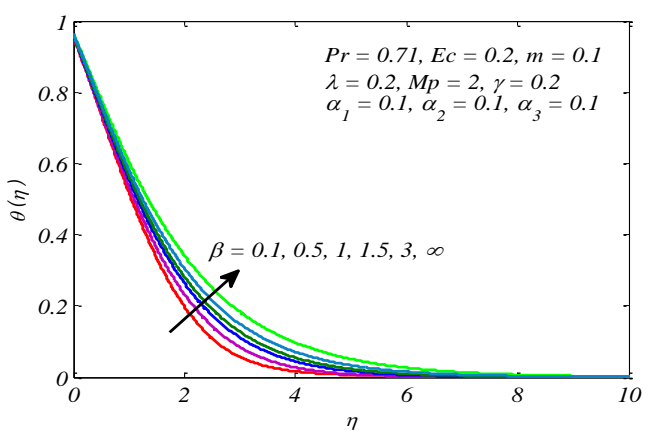

Fig. 3: Dimensionless temperature profiles $\theta(\eta)$ for the various values of $\beta$.

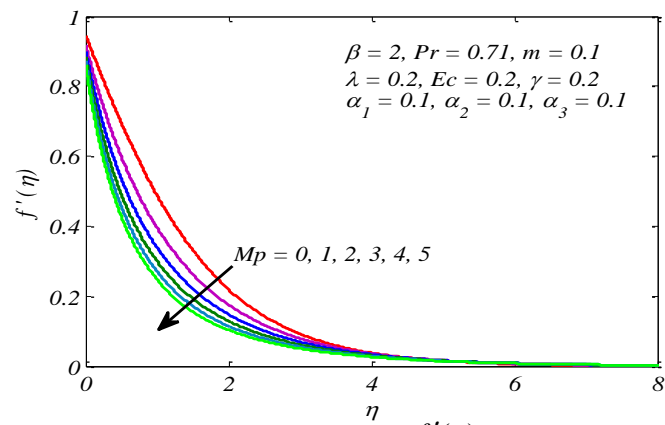

Fig. 4: Dimensionless velocity profiles $f^{\prime}(\eta)$ for the various values of Mp

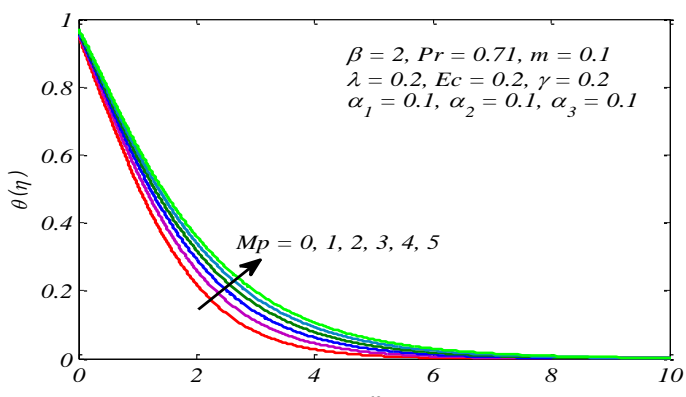

Fig. 5: Dimensionless temperature profiles $\theta(\eta)$ for the various values of $M p$. 


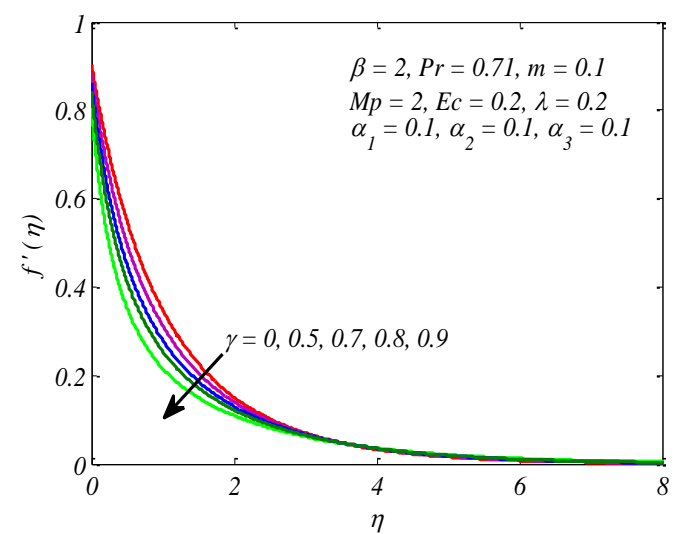

Fig. 6: Dimensionless velocity profiles $f^{\prime}(\eta)$ for the various values of $\gamma$.

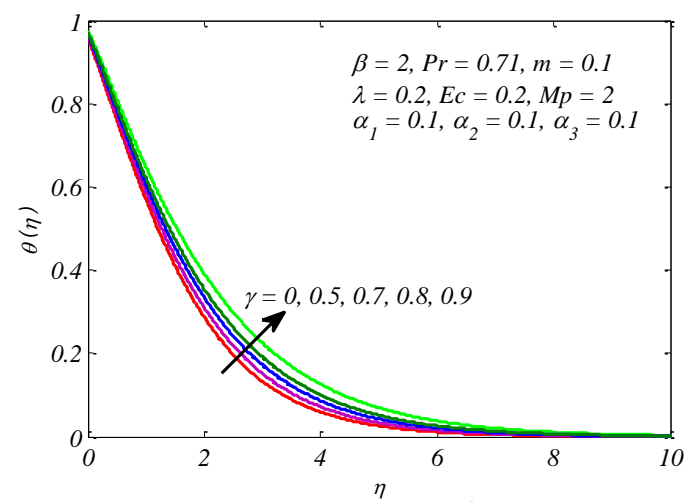

Fig. 7: Dimensionless temperature profiles $\theta(\eta)$ for the various values of $\gamma$.

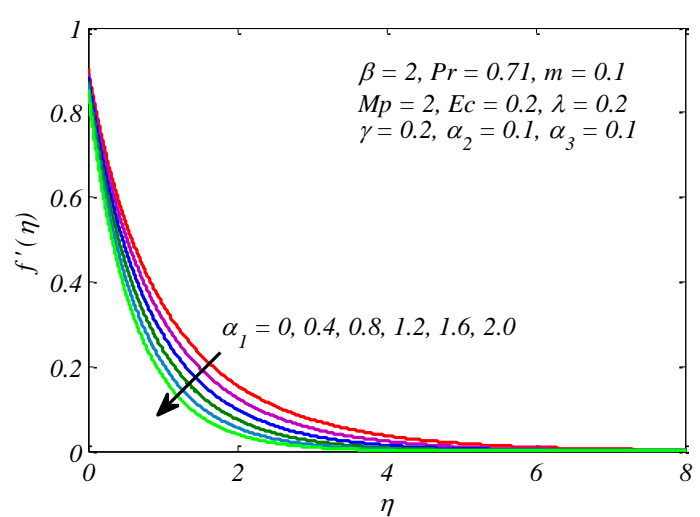

Fig. 8a: Dimensionless velocity profiles $f^{\prime}(\eta)$ for the various values of $\alpha_{1}$ for $m<1$.

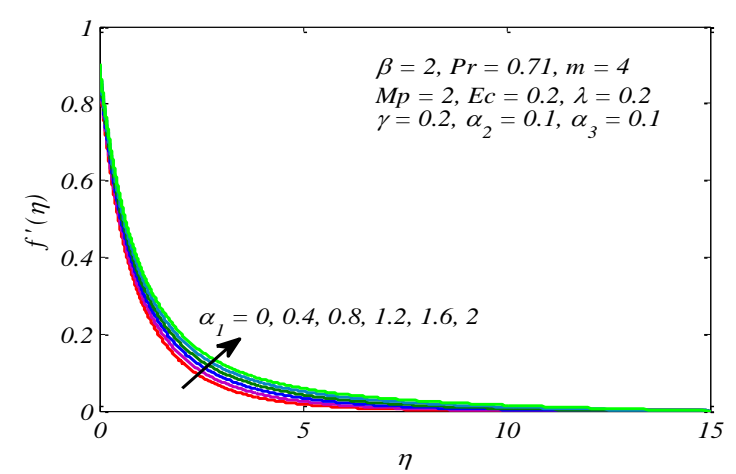

Figure 8b: Dimensionless velocity profiles $f^{\prime}(\eta)$ for the various values of $\alpha_{1}$ for $m>1$.

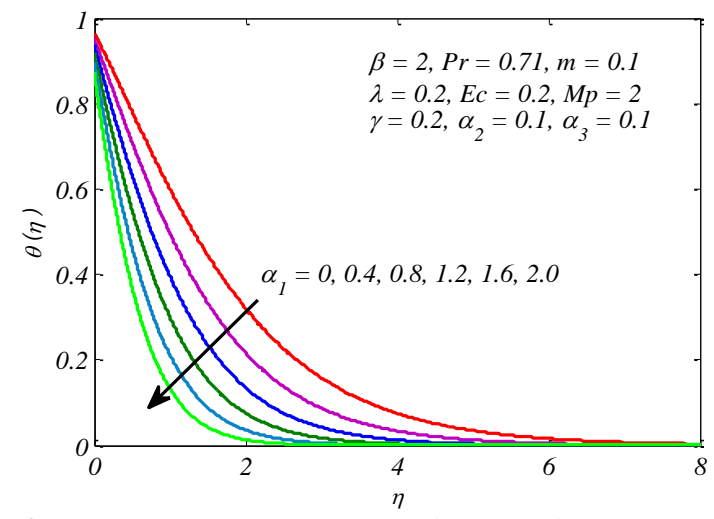

Fig. 9a: Dimensionless temperature profiles $\theta(\eta)$ for the various values of $\alpha_{1}$ for $m<1$.

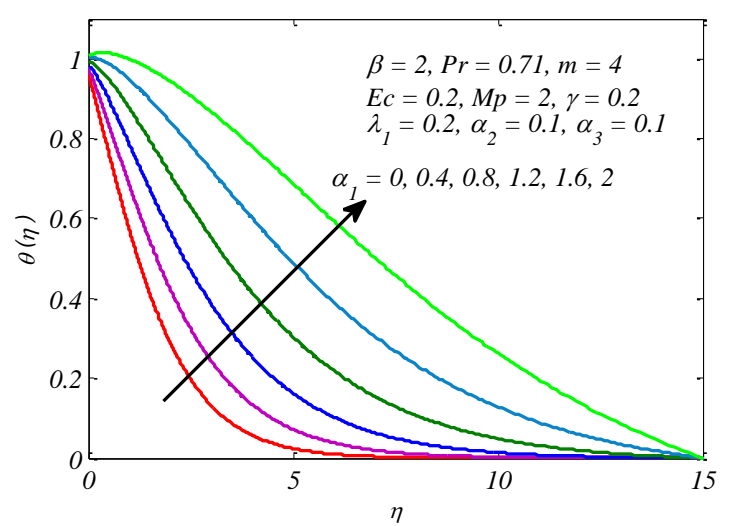

Fig. 9b: Dimensionless temperature profiles $\theta(\eta)$ for the various values of $\beta$ for $m>1$.

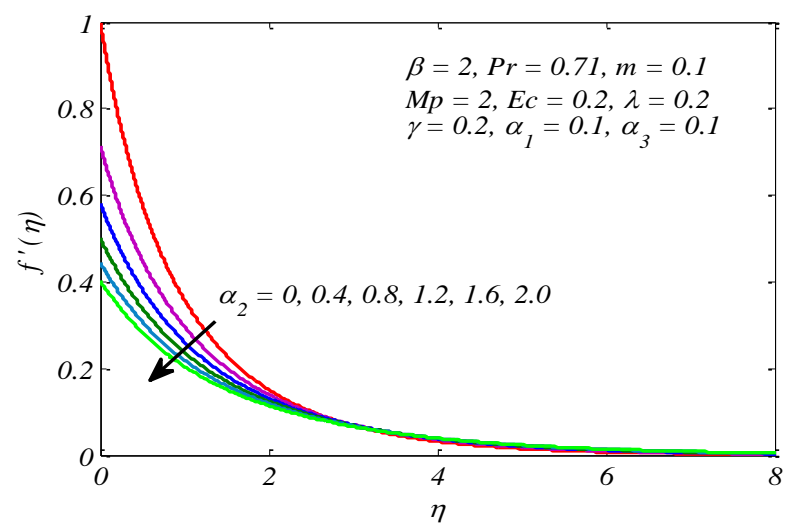

Fig. 10: Dimensionless velocity profiles $f^{\prime}(\eta)$ for the various values of $\alpha_{2}$.

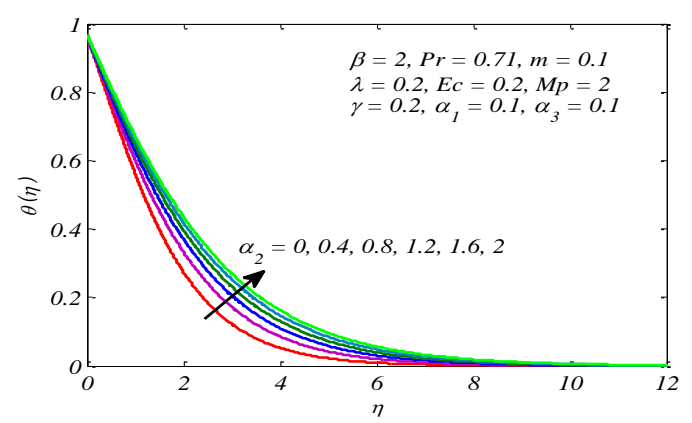

Fig. 11: Dimensionless temperature profiles $\theta(\eta)$ for the various values of $\alpha_{2}$. 


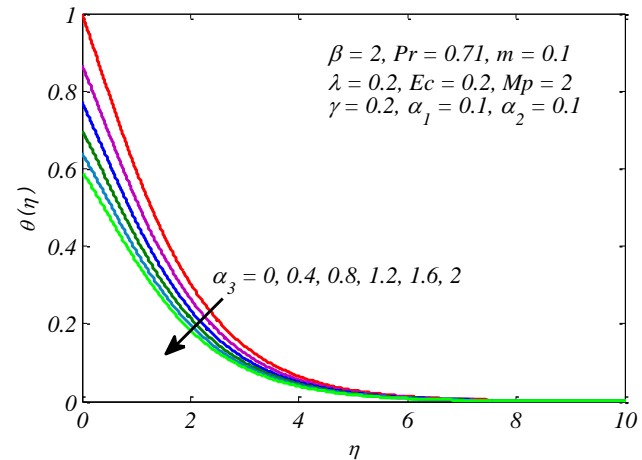

Fig. 12: Dimensionless temperature profiles $\theta(\eta)$ for the various values of $\alpha_{3}$.

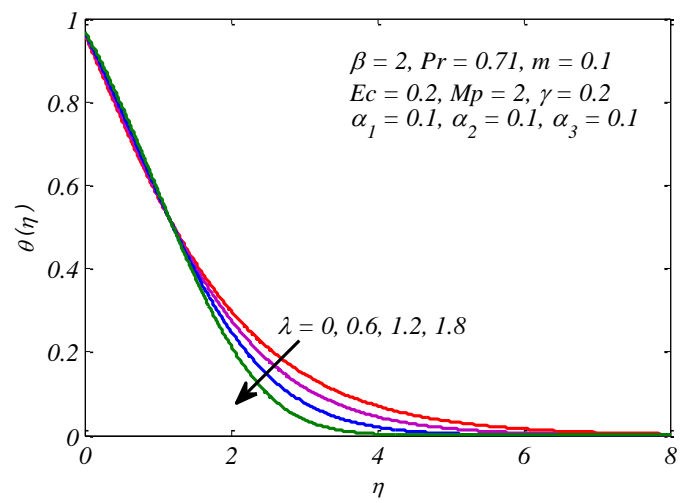

Fig. 13: Dimensionless temperature profiles $\theta(\eta)$ for the various values of $\lambda$.

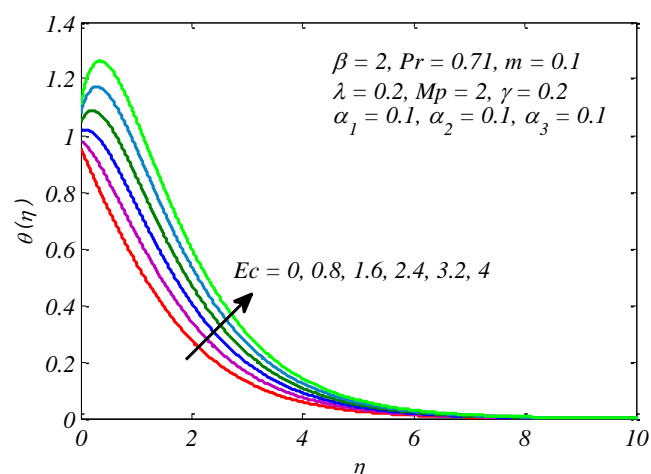

Figure 14: Dimensionless temperature profiles $\theta(\eta)$ for the various values of $E c$.

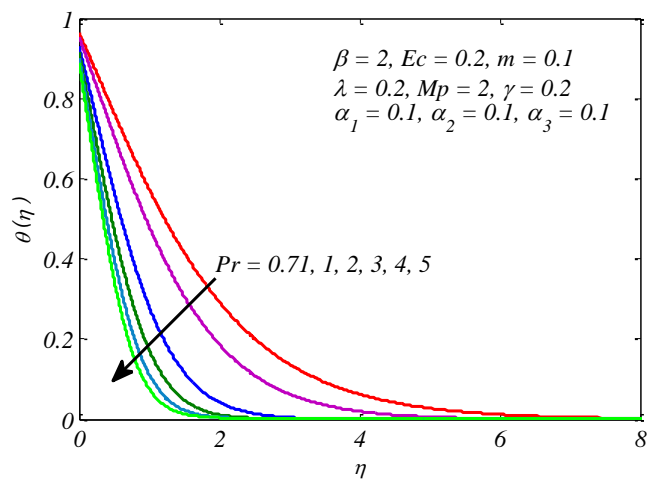

Fig. 15: Dimensionless temperature profiles $\theta(\eta)$ for the various values of $\operatorname{Pr}$.

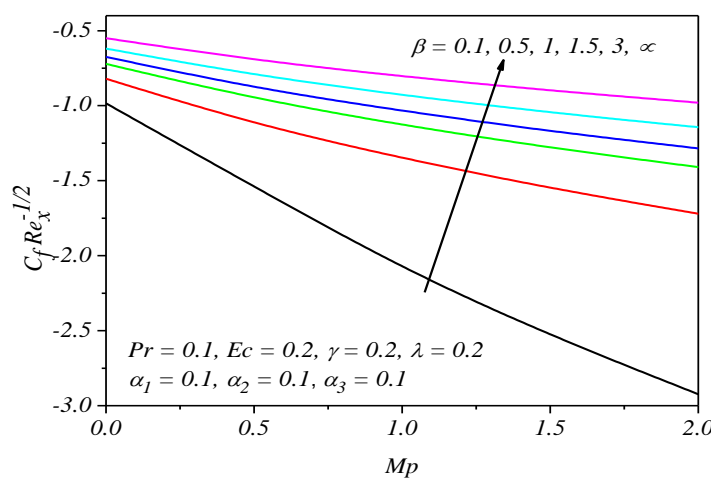

Fig. 16: Skin friction coefficient $C_{f} \operatorname{Re}_{x}^{1 / 2}$ for the various values of $M p$ and $\beta$.

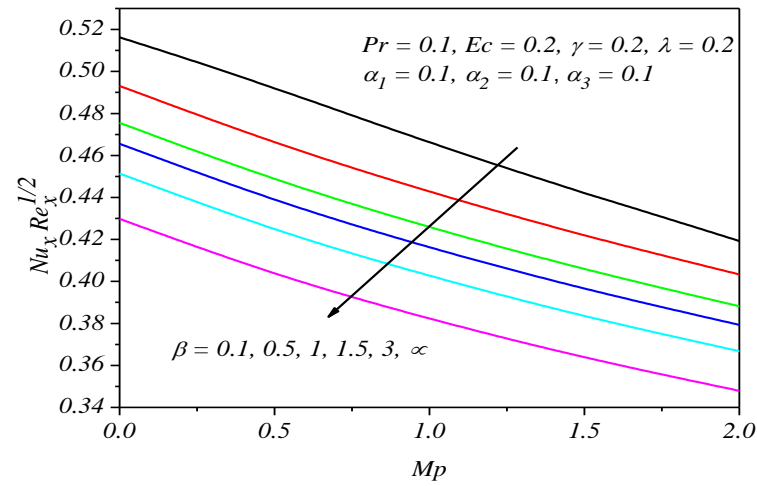

Fig. 17: local Nusselt number $N u_{x} \operatorname{Re}_{x}^{-1 / 2}$ for the various values of $M p$ and $\beta$.

Table 1: Comparison of skin friction coefficient for different values of $M$ when $\gamma=0$.

\begin{tabular}{|l|c|c|c|c|}
\hline \multicolumn{1}{|c|}{$P r$} & Wang [46] & $\begin{array}{c}\text { Salahuddin et } \\
\text { al. [47] }\end{array}$ & $\begin{array}{c}\text { Malik et al. } \\
{[48]}\end{array}$ & Present results \\
\hline 0.07 & 0.0656 & 0.0654 & 0.0651 & 0.0656225696 \\
0.2 & 0.1691 & 0.1688 & 0.1683 & 0.1690885738 \\
0.7 & 0.4539 & 0.4535 & 0.4537 & 0.4539161580 \\
2.0 & 0.9114 & 0.9108 & 0.9109 & 0.9113576837 \\
7.0 & 1.8954 & 1.8944 & 1.8948 & 1.8954032582 \\
20.0 & 3.3539 & 3.3522 & 3.3521 & 3.3539041437 \\
70.0 & 6.4622 & 6.4619 & 6.4626 & 6.4621995452 \\
\hline
\end{tabular}

\section{Conclusion}

This paper numerically studied the slip flow of a viscous dissipative Casson fluid with Cattaneo-Christov heat flux model in the presence of variable viscosity. Some important results of our investigation are given below:

- Hydrodynamic boundary layer is thinner in Casson fluid when compared with the viscous fluid.

- The energy dissipation is due to heat, viscous dissipation and deformation work has the effect to thicker the thermal boundary layer increases in the temperature profile, and hence decreases the heat transfer rate from the surface.

- The temperature distribution is higher to the slip boundary flow than to the no-slip boundary layer flow, but this behavior is contrary for the velocity field.

- Temperature distribution increases more rapidly in Fourier's law case compared to Cattaneo-Christov heat flux model.

- The absolute value of Nusselt number and skin friction coefficient decreases for the higher values of Casson fluid parameter. 


\section{Nomenclature}

$c_{p}$ - Specific heat at constant pressure $(\mathrm{J} / \mathrm{kg} K)$

F- Dimensionless stream function

$u$ - Velocity component in X-direction $(\mathrm{m} / \mathrm{s})$

$v$ - Velocity component in y-direction $(\mathrm{m} / \mathrm{s})$

$T$ - Temperature of the fluid $\left({ }^{0} \mathrm{C}\right)$

$T_{w}$ - Temperature at the stretching surface

$T_{\infty}$ - Ambient fluid temperature

$\mathrm{U}_{0}$ - Reference velocity

$\mathrm{P}_{\mathrm{z}}-$ Yield stress on the field

$M p$ - Hartmann number

$E c$ - Eckert number

I - Identity Matrix

[ ] - Diagonal Matrix

$\mathcal{D}$ - Differentiation matrix

$\varphi$ - Dissipative function

$B_{0}$ - Magnetic field strength

$\mathrm{q}$ - Heat flux

$\lambda_{2}-$ Thermal relaxation time

$L$ - Scale

$\mathrm{L}_{1}-$ Slip velocity coefficient

$t$ - Time

$\mathrm{N}-$ Number of grid points

$\mathrm{Pr}$ - Prandtl number

$C_{f}$ - Skin friction coefficient

$\mathrm{D}_{1}-$ Temperature jump coefficient

$\alpha_{1}$ - The plate surface

$\alpha_{2}$ - Slip velocity factor

$\alpha_{3}$ - Thermal jump factor

Greek symbols

$\kappa$ - Thermal conductivity (W/m K)

$\mu$ - Thermal viscosity $(N \mathrm{~s} / \mathrm{m})$

$\rho$-Fluid density $\left(\mathrm{kg} / \mathrm{m}^{3}\right)$

$\gamma$ - The variable viscosity

$\lambda$ - Thermal relaxation parameter

$\tau_{w}$ - Wall shear stress

$\mu_{\beta}$ - Plastic dynamic viscosity of

\section{Non-newtonian fluid}

$v=\mu / \rho$ - Kinematic viscosity of the fluid

$\eta$-Similarity variable

$\tau$ - Extra stress tensor

$\beta$ - Cason parameter

$\theta$ - Non-dimensional temperature

\section{Subscript}

$w$-Condition at the surface

$\infty$ - Condition at infinity

$\mathrm{r}$ - Iteration number

\section{Super script}

$\mathrm{p}$ - Differentiation with respect to $\eta$

\section{References}

[1] Crane, L J (1970), Flow past a Stretching Plate, Zeit. Ang.Mat Phys. 21(4), 645-647.

[2] Pavlov, K B (1974), Magnetohydrodynamic Flow of an In compressible Viscous Fluid Caused by the Deformation of a Plane Surface, Magnetohydr. 10, 146-148.
[3] Gupta, P S \& Gupta, A S (1977), Heat and Mass Transfer on a Stretching Sheet with Suction or Blowing, Canadian J. Chem. Eng., 55( 6), 744-746.

[4] Fourier, J B J (1822), Théorie Analytique De La Chaleur, Paris.

[5] Cao, B Y, Guo, Z Y (2007), Equation of motion of a phonon gas and non-Fourier heat conduction. Journal of Applied Physics 102, 053503.

[6] Dong, Y, Cao, B Y \& Guo, Z Y (2011), Generalized heat conduction laws based on thermomass theory and phonon hydrodynamics. Journal of Applied Physics 110, 063504.

[7] Zhang, M K, Cao, B Y \& Guo, Y C (2013), Numerical studies on dispersion of thermal waves, International Journal of Heat and Mass Transfer 67, 1072-1082.

[8] Cattaneo, C (1948), Sulla conduzione del calore, Atti Semin. Mat. Fis. Univ. Modena Reggio Emilia 3, 83-101.

[9] Christov, C I (2009), On frame indifferent formulation of the Maxwell - Cattaneo model of finite-speed heat conduction. Mechanics Research Communications 36. 481-486.

[10] Straughan, B (2010) Thermal convection with the CattaneoChristov model, International Journal of Heat and Mass Transfer 53 , 95-98.

[11] Ciarletta, M \& Straughan, B (2010), Uniqueness and structural stability for the Cattaneo-Christov equations, Mechanics Research Communications 37, 445-447.

[12] Han, S, Zheng, L, Li, C \& Zhang, X (2014), Coupled flow and heat transfer in viscoelastic fluid with Cattaneo-Christov heat flux model, Applied Mathematics Letters 38, 87-93

[13] Khan, A J, Mustafa, M, Hayat, T \& Alsaedi, A (2015), Numerical Study of Cattaneo-Christov Heat Flux Model for Viscoelastic Flow Due to an Exponentially Stretching Surface.PLoS ONE, 10(9), e0137363. doi:10.1371/journal.pone.0137363

[14] Malik, M Y, Mair Khan, Salahuddin, T \& Imad Khan, (2016), Variable viscosity and MHD flow in Casson fluid with CattaneoChristov heat flux model: Using Keller box method, Engineering Science and Technology, an International Journal 19, 1985-1992

[15] Hayat, T, Qayyum, S, Imtiaz, M \& Alsaedi A (2017), Flow between two stretchable rotating disks with Cattaneo-Christov heat flux model, Results in Physics 7, 126-133

[16] Hayat, T, Khan, M I, Farooq, M \& Alsaedi A (2017), Thermally stratified stretching flow with Cattaneo-Christov heat flux, International Journal of Heat and Mass Transfer 106, 289-294.

[17] Casson, N., Rheology of Disperse Systems, in Flow Equation

[18] for Pigment Oil Suspensions of the Printing Ink Type. Rheo gy of Disperse Systems, Mill, C.C., Ed., London: Pergamon, 1959, 84 102.

[19] S. Nadeem, R U Haq, C. Lee C (2012), MHD flow of a Casson fluid over an exponentially shrinking sheet, Sci. Iran. 19, 1550-1553.

[20] Mukhopadhyay, S, De, P R, Bhattacharyya, K \& Layek, G C (2013), Casson fluid flow over an unsteady stretching surface, Ain Shams Eng. J. 4, 933-938.

[21] Mukopadhyay, S (2013), Casson fluid flow and heat transfer over a nonlinearly stretching surface, Chin. Phys. 22, 074701.

[22] Nadeem, S, Haq, R U, Akbar, N S, Khan, Z H (2013), MHD threedimensional Casson fluid flow past a porous linearly stretching sheet, Alexandria Eng. J. 52, 577-582.

[23] Mukhopadhyaya, S, Moindala, I C \& Hayat, T (2014), MHD boundary layer flow of Casson fluid passing through an exponentially stretching permeable surface with thermal radiation, Chin. Phys. 23, 104701.

[24] Mahanta, G \& Shaw, S (2014), 3D Casson fluid flow past a porous linearly stretching sheet with convective boundary condition, AlexandriaEng.J., http//dx.doi.org/10.1016/j.aej.2015.04.014

[25] Mustafa, M \& Khan, J A (2015), Model for flow of Casson nanofluid past a non-linearly stretching sheet considering magnetic field effects, AIP Adv. 5, 077148.

[26] Animasaun, I L, Adebile, E A \& Fagbade, A I (2015), Casson fluid flow with variable thermo- physical property along Exponentially stretching sheet with suction and exponentially decaying internal heat generation using the homotopic analysis Method, J. Niger. Math Soc., http://dx.doi.org/10.1016/j.jnnms. 2015.02.001.

[27] Das, M, Mahato, R \& Nandkeolyar, R (2015), Newtonian heating effect on unsteady hydro- magnetic Casson fluid flow past a flat plate with heat and mass transfer, Alexandria Eng. J., http://dx.doi/10.1016/j.aej.2015.07.007.

[28] Raju, C S K, Sandeep, N, Sugunamma, V, Babu, M J \& Reddy, J V $\mathrm{R}$ (2016), Heat and mass transfer in magnetohydrodynamic Casson fluid over an exponentially permeable stretching surface, Eng. Sci. Technol. Int. J. 19 (1), 45-52. 
[29] Ramesh, K \& Devakar, M (2015), Some analytical solutions for flows of Casson fluid with slip boundary conditions, Ain Shams Eng. J. 6, 967-975.

[30] Khalid, A, Khan, I, Khan, A \& Shafie, S (2015), Unsteady MHD free convection flow of Casson fluid past over an oscillating vertical plate embedded in a porous medium, Eng. Sci.Technol. Int. J. 18 (3),309-317.

[31] Ibrahim S M, Kumar P V, Lorenzini G, Lorenzini E, \& Mabood, F (2017), Numerical Study of the Onset of Chemical Reaction and Heat Source on Dissipative MHD Stagnation Point flow of Casson Nanofluid over a Nonlinear Stretching Sheet with Velocity Slip and convective Boundary Conditions, Journal of Engineering Thermophysics 26(2), 256-271.

[32] Seth, G S, Tripath, R \& Mishra, M K (2017), Hydromagnetic thin film flow of Casson fluid in non- darcy porous medium with Joule dissipation and Navier's partial slip, Appl. Math. Mech. -Engl. Ed. DOI 10.1007/s10483-017-2272-7

[33] Shateyi, S, Mabood, F \& Lorenzini, G (2017), Casson Fluid Flow: Free Convective Heat and Mass Transfer over an Unsteady Permeable Stretching Surface Considering Viscous Dissipation, Journal of Engineering Thermophysics 26(1), 39-52.

[34] B. Gebhart, (1962), Effects of viscous dissipation in natural convection, J. Fluid Mech. 14, 225-232.

[35] Turcotte, D, Hsui, A, Torrance, K \& Schubert, G (1974), Influence of viscous dissipation on B'enard convection, J. Fluid Mech. 64 369-374.

[36] Barletta, A, Celli, M \& Rees, D (2009), The onset of convection in a porous layer induced by viscous dissipation:Alinear stability analysis, Int. J. Heat Mass Transfer 52, 337.

[37] Barletta, A, Celli, M \& Nield, D (2010),Unstably stratified Darcy flow with impressed horizontal temperature gradient, viscous dissipation and asymmetric thermal boundary conditions, Int. J. Heat Mass Transfer 53, 1621-1627.

[38] Barletta, A \& Nield, D (2010), Instability of Hadley-Prats flow with viscous heating in a horizontal porous layer, Transp. Porous Media 84, 241-256.

[39] Barletta, A \& Nield, D (2011), Thermosolutal convective instability and viscous dissipation effect in a fluid-saturated porous medium," Int. J. Heat Mass Transfer 54, 1641-1648.

[40] Roy, K \& Murthy, P, Soret effect on the double diffusive convection instability due to viscous dissipation in a horizontal porous channel, Int. J. Heat Mass Transfer 91, 700-715).

[41] Khader, M M \& Mziou, S (2017), Chebyshev spectral method for studying the viscoelastic slip flow due to a permeable stretching surface embedded in a porous medium with viscous Dissipation and non-uniform heat generation, Boundary Val ue Problems 2017:37, DOI 10.1186/s13661-017-0764-2

[42] Roy, K \& Murthy, PVSN (2017), Effect of viscous dissipation on the convective instability induced by inclined temperature gradients in a non-Darcy porous medium with horizontal through flow, Physics of Fluids 29, 044104; doi: 10.1063/1.4979526

[43] Metri, P G, Guariglia, E, \& Silvestrov, S (2017) Lie group analysis for MHD boundary layer flow and heat transfer over stretching sheet in presence of viscous dissipation and uniform heat source/sink, AIP Conference Proceedings 1798, 020096 doi: 10.1063/1.4972688

[44] Palani, G, Srikanth, U, \& Kim, K Y, (2017) Combined Effects of Viscous Dissipation and MHD on Free Convection Flow past a Semi-Infinite Vertical Plate with Variable Surface temperature in the Presence of Heat Source, Journal of Engineering Thermophysics, 26,113-124.

[45] Motsa S S \& Makukula Z G, (2013), On spectral relaxation method approach for steady von kárman flow of a reiner-rivlin fluid with joule heating, viscous dissipation and suction/injection. Cent. Eur. J. Phys., 11(3), 363-374.

[46] Kameswaran, P, Sibanda, P, \& Motsa, S S, (2013), A spectral relaxation method for thermal dispersion and radiation effects in a nanofluid flow, Boundary Value Problems 2013, 242.

[47] Wang, C Y (1989), Free convection on a vertical stretching surface with suction and blowing, Appl. Math. Mech. 69, 418-420.

[48] Salahuddin, T, Malik, M Y, Hussain, A, Bilal, S \& Awais, M (2016), MHD flow of Cattaneo- christov heat flux model for Williamson fluid over a stretching sheet with variable thickness: using numerical approach, J. Magn. Magn. Mater. 401, 991-997.

[49] Malik, M Y, Khan, M, Salahuddin, T \& Khan I (2016), Variable viscosity and MHD flow in casson fluid with Cattaneo-Christov heat flux model: Using Keller box method, Engineering Science and Technology, an International Journal 19, 1985-1992. 